\title{
Zaubernussgewächse (Hamamelidaceae) - nicht nur Hamamelis
}

\author{
VeIT DÖRKEN \& ANNETTE HÖgGEMEIER
}

\begin{abstract}
The Hamamelidaceae are known since the late Cretacecous. Today the family has a wide but disjunct distribution with the greatest diversity in Middle and South China. The species which are hardy in Central Europe are important trees and shrubs for solitary arrangements in gardens and parks. Most of them flower in a spectacular way during late winter and early spring and produce a bright autumn colour.
\end{abstract}

\section{Zusammenfassung}

Die Hamamelidaceen sind eine erdgeschichtlich alte Familie, die fossil seit der späten Kreidezeit bekannt ist. Heutzutage hat die Familie ein weites, jedoch disjunktes Verbreitungsareal mit einem Verbreitungsschwerpunkt in Mittel- bis Süd-China. Zahlreiche der unter mitteleuropäischem Klima im Freiland kultivierbaren Arten sind aufgrund des frühen Blütezeitpunktes sowie der spektakulären Herbstfärbung wichtige Blatt- und Blütenschmuckgehölze.

\section{Systematik}

Derzeit werden zu den Zaubernussgewächsen rund 100 Arten aus 30 Gattungen gestellt, wobei rund die Hälfte der Gattungen wie z. B. Disanthus, Parrotia, Parrotiopsis und Sycopsis heute monotypisch sind; fossil sind sie z. T. aber auch mit mehreren Arten vertreten.

\section{Verbreitung}

Die Zaubernussgewächse weisen heute ein großes Areal auf, das allerdings unterbrochen ist. Der Verbreitungsschwerpunkt liegt in subtropischen bis gemäßigten Arealen Mittel- bis Süd-Chinas. Sie kommen darüber hinaus vereinzelt auch in Malaysien, Australien und in Kleinasien sowie im tropischen Ost- bis SüdAfrika und Madagaskar vor. Einige sind auch in Nord- und Mittel-Amerika vertreten (ENGLER 1964). Die Familie ist bereits aus der späten Kreidezeit bekannt (TAYLOR et al. 2009) und hatte ehemals ein wesentlich größeres Verbreitungsgebiet (LEHMANN et al. 1978, MAI 1995). Mit einigen Gattungen war sie auch in Mitteleuropa heimisch (FERGUSON \& KNOBLOCH 1998). Liquidambar, Hamamelis und Fothergilla waren im Tertiär in einem ausgedehnten arktischen eurasisch-nordamerikanischen Gebiet heimisch (FUKAREK 2000). Ein weiteres, aus der arktotertiären Vegetation stammendes Relikt ist die Gattung Parrotia (SCHMIDT 2005).

\section{3. Äußeres Erscheinungsbild}

Die meisten Hamamelidaceen sind immergrüne oder winterkahle Großsträucher bis kleine Bäume. Einige Arten wie z. B. der 30 (-45) m hohe Amberbaum (Liquidambar styraciflua) sind Großbäume mit vielgestaltigen Kronenformen. Die Großsträucher wachsen oft breit trichterförmig. Amberbäume sind sparrig bis steiftriebig verzweigt. Anderen Habitus zeigt die Armblütige Scheinhasel (Corylopis pauciflora), die eher locker und dünntriebig wächst.

Die Überwinterungsknospen der Hamamelidaceen sind recht unterschiedlich gestaltet. $\mathrm{Co}$ rylopsis und Liquidambar haben Winterknospen mit echten Knospenschuppen. Bei Fothergilla, Hamamelis, Parrotia und Sinowilsonia dagegen übernehmen kleine Laubblätter den Schutz des Vegetationspunktes, wie es auch vom Wolligen Schneeball (Viburnum lanata) bekannt ist. Diese Art des Knospenschutzes deutet in der Regel auf einen subtropischen bis tropischen Ursprung der entsprechenden Gruppe hin.

Hamamelidaceen umfassen laubabwerfende sowie immergrüne Arten mit wechselständigen Blättern. Die nachfolgend vorgestellten Arten sind alle laubabwerfend, die immergrünen Vertreter sind für die mitteleuropäische Freilandkultur nicht bzw. nur lokal geeignet. Alle laubabwerfenden Zaubernussgewächse verfügen über eine prächtige Herbstfärbung von Hell- bis Goldgelb über Orange bis leuchtend Rot. Einige Arten färben ungewöhnlich pflaumenblau aus. 


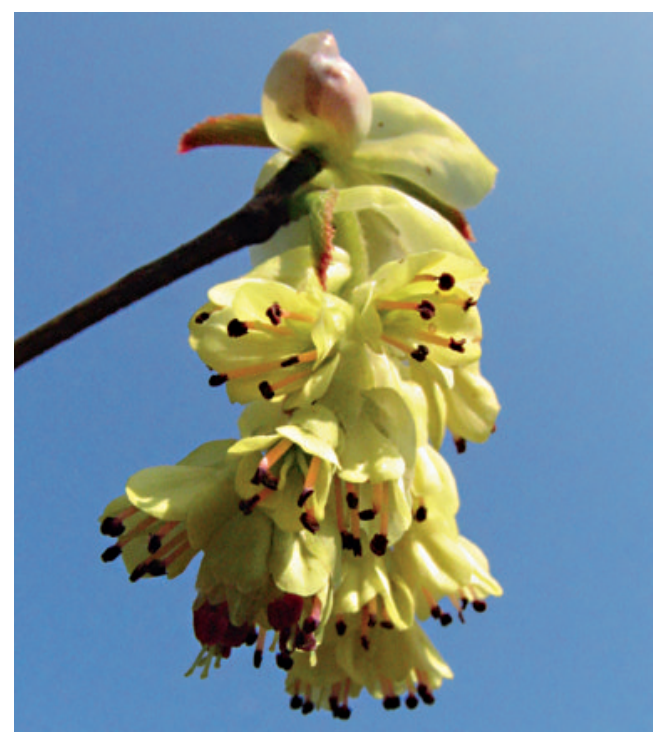

Die Blüten sind eingeschlechtlich (z. B. Liquidambar) oder zwittrig (z. B. Hamamelis). Bei vielen Arten ist die Blütenhülle stark reduziert, nicht selten fehlen Kelch- und Kronblätter ganz. Bei den meisten dieser laubabwerfenden Hamamelidaceen erfolgt die Blüte vor dem Laubaustrieb. Hamamelis vernalis, die zum Zeitpunkt des Laubabwurfs blüht, stellt innerhalb der Gattung eine Ausnahme dar. Das Farbspektrum der Hamamelidaceen-Blüten reicht von Weiß (z. B. Fothergilla) über Gelb (z. B. Hamamelis mollis) bis zu Orange und Dunkelrot (Disanthus). Bei vielen Arten verströmen die Blüten einen intensiven, süßlichen Duft.

Die Früchte sind verholzende Kapseln, die sich entlang der Verwachsungsnaht der Fruchtblätter meist nur im oberen Viertel öffnen, um die Samen zu entlassen. Je Kapselfach sind z. B. bei Liquidambar mehrere Samen enthalten, bei Fortunearia wird nur ein einziger, teilweise geflügelter Same entwickelt.

\section{Verwendung}

Nur wenige Arten sind von forstwirtschaftlicher Bedeutung (z. B. Liquidambar). Von anderen werden die Inhaltsstoffe für Kosmetika und pharmazeutische Produkte genutzt (z. B. Hamamelis). Zahlreiche Arten haben aufgrund ihres hohen Zierwertes den Eingang in die mitteleuropäische
Gartenkultur gefunden. Bei uns findet man nur ein vergleichsweise geringes HamamelidaceenSortiment, das winterhart und somit auch für die Freilandkultur geeignet ist. Die nachfolgend beschriebenen Arten sind in mitteleuropäischen Gärten mehr oder weniger häufig anzutreffen und auch im deutschen Baumschulhandel verfügbar. Es handelt sich um Gehölze für Einzelpflanzungen in kleineren und größeren Hausgärten sowie Parkanlagen. Viele dieser Arten stellen wertvolle Habitus-, Borken-, Blatt- und Blütenschmuckgehölze dar.

\section{Zaubernussgewächse in unseren Gärten}

\subsection{Corylopsis (Scheinhasel, Glockenhasel)}

Corylopsis-Arten erinnern im nichtblühenden Zustand an Haselsträucher, worauf sich auch die deutschen Namen beziehen. Ebenso verweist die botanische Bezeichnung Corylopsis (von lat. corylus = Hasel und griech . opsis = Aussehen) auf einen haselähnlich aussehenden Strauch. Dabei erfolgte die Benennung nicht in Anlehnung an die heimische Gewöhnliche Hasel (Corylus avellana), sondern an die Japanische Hasel (C. sieboldiana). Scheinhaseln sind jedoch nicht näher mit echten Haseln (Birkengewächsen) verwandt. Corylopsis umfasst 7 (MABBERLEY 2008) winterkahle Arten, die im östlichen Himalaja, China und Japan heimisch sind. Scheinhaseln erfreuen sich aufgrund der in der Regel unkomplizierten Kultur auch in mitteleuropäischen Gärten einer großen Beliebtheit als Blüten- und Blattschmucksolitäre. Sie benötigen für ein optimales Gedeihen einen sonnigen bis leicht schattigen, tiefgründigen Standort mit saurer bis neutraler Bodenreaktion. Scheinhaseln kommen vor ruhigen oder dunklen Hintergründen, z. B. aus Bambus, Koniferen, Rhododendron oder auch vor dunklen Hauswänden, sehr gut zur Geltung. Besonders reizvoll sind Scheinhaseln in Kombination mit blau blühenden Frühlingsgeophyten wie Scilla oder Muscari (DÖrken \& HöGGEMEIER 2009). In sehr strengen Wintern kann es zum Zurückfrieren junger Triebe kommen. Darüber hinaus gelten sie als tendenziell spät-

Abb. 1: Corylopsis pauciflora. 
frostgefährdet (Kelly \& Hillier 2004). Die sattbis hellgelben, zwittrigen Blüten sind fünfzählig und erscheinen stets vor dem Laubaustrieb. Scheinhaseln stellen somit für den Vorfrühlingsbis Frühlingsaspekt eine elegante Alternative zu den überreichlich gepflanzten Forsythien dar. Die beiden nachfolgend beschriebenen Arten gehören zum Standartsortiment deutscher Baumschulen.

Corylopsis pauciflora (Armblütige Scheinhasel) stammt aus den Gebirgen Japans und Taiwans und ist ein bis $2 \mathrm{~m}$ hoher winterkahler, feingliedrig verzweigter Strauch. Er wurde bereits um 1860 nach Europa eingeführt. Die Armblütige Scheinhasel hat, wie alle anderen Scheinhaseln auch, echte Winterknospen. Die eiförmigen Blätter werden bis $6 \mathrm{~cm}$ lang und haben eine deutlich herzförmige Basis. Der Blattrand ist buchtig gezähnt, wobei jeder Zahn in eine kleine Grannenspitze ausläuft. Nerven sowie Blattrand sind auf der Blattunterseite leicht weißlich behaart. Die meist zu 2 bis 3, selten zu mehreren zusammenstehenden hellgelben, nach Schlüsselblumen duftenden Blüten bilden kurze herabhängende Ähren. Die volkstümliche Bezeichnung Armblütige Scheinhasel bezieht sich auf die im Vergleich zu den übrigen Scheinhaseln wenigen, meist nur 3 bis 4 Einzelblüten je Blütenstand, die von März bis April vor dem Laubaustrieb erscheinen. Scheinhaseln sind dementsprechend wichtige Vorfrühlingsblüher, die eine gute Bienen- und Hummelweide darstellen. Die zweifächerigen Kapseln sind bis $0,8 \mathrm{~cm}$ dick, öffnen sich zweiklappig und sind stark gehörnt, da die Griffel an der Frucht erhalten bleiben. Im Herbst färben sich die Blätter Hell- bis Zitronengelb. Die Art wurde bereits mehrfach als besonders attraktives Gartengehölz ausgezeichnet.

Corylopsis spicata (Ährige Scheinhasel) wird bis 2,5 m hoch. Sie stammt ebenfalls aus Japan sowie der chinesischen Provinz Kiangsi. In ihrer Heimat wächst sie auf bodenfeuchten Standorten und leidet daher bei uns in Trockenperioden besonders unter Bodentrockenheit und Hitze. Für ein optimales Gedeihen sollte dementsprechend auf eine gleichmäßige Feuchtigkeitsversorgung geachtet werden. Auch sie wurde schon um 1860 nach Mittel-Europa eingeführt. In Japan ist sie ein beliebter und häufig kultivierter Solitärstrauch. Seine Triebe sind wesentlich derber als bei der vorherigen Art und zunächst deutlich behaart. Die breit-eiförmigen bis länglich-eiförmigen Blätter werden bis $15 \mathrm{~cm}$ lang und sind buchtig gezähnt. Die Blattunterseite ist anfangs leicht behaart, später kahl. Im Herbst zeigt sich eine kräftig goldgelbe Laubfärbung. Die leuchtend gelben Blüten befinden sich zu mehreren in bis zu $7 \mathrm{~cm}$ langen, 6 bis 12-blütigen, herabhängenden Ähren. Der Blühzeitraum von März bis teilweise noch in den Mai hinein ist recht lang. Die sich zweiklappig öffnenden, zweifächerigen Kapseln werden bis $1,2 \mathrm{~cm}$ dick und sind durch die bleibenden Griffel stark gehörnt.

In den Sortimentskatalogen einiger Spezialbaumschulen werden noch weitere CorylopsisArten angeboten, die auch in der mitteleuropäischen Gartenkultur aufgrund des hohen Zierwertes größere Beachtung verdienen wie C. glabrescens (Blüten schwefelgelb, in $5-6 \mathrm{~cm}$ langen hängenden Ähren, duftend, April); C. veitchii (Blüte gelblichgrün bis satt gelb, in vielblütigen bis $5 \mathrm{~cm}$ langen Ähren, duftend, April); C. willmottiae (Blüten hell- bis blassgelb, in vielblütigen bis zu $8 \mathrm{~cm}$ langen Ähren, duftend, Ende März-April).

\subsection{Disanthus cercidifolius (Doppelblüte)}

Eine wenig bekannte und selten kultivierte Gattung ist die Doppelblüte. Die volkstümliche Bezeichnung Doppelblüte ist die wörtliche Übersetzung des Gattungsnamens (von gr. dis = doppelt und anthos = Blüte). Die Gattung Disanthus umfasst nur eine Art. Sie stammt aus den Hochgebirgen Honshus in Japan, wo sie an halbschattigen Standorten auf feuchten, gut durchlässigen Böden mit saurer Bodenreaktion als Großstrauch bis $4(-5) \mathrm{m}$ hoch wird. Die Verzweigung ist fein und aufsteigend. Daher ist der Habitus dieses winterkahlen Strauches mehr oder weniger trichterförmig und erinnert somit an Scheinhaseln (Corylopsis). Die Triebe sind dicht mit hellen Korkwarzen besetzt. Ihre rötlichen, länglich eiförmigen Winterknospen sind kahl. An den Zweigen befinden sich in wechselständiger 

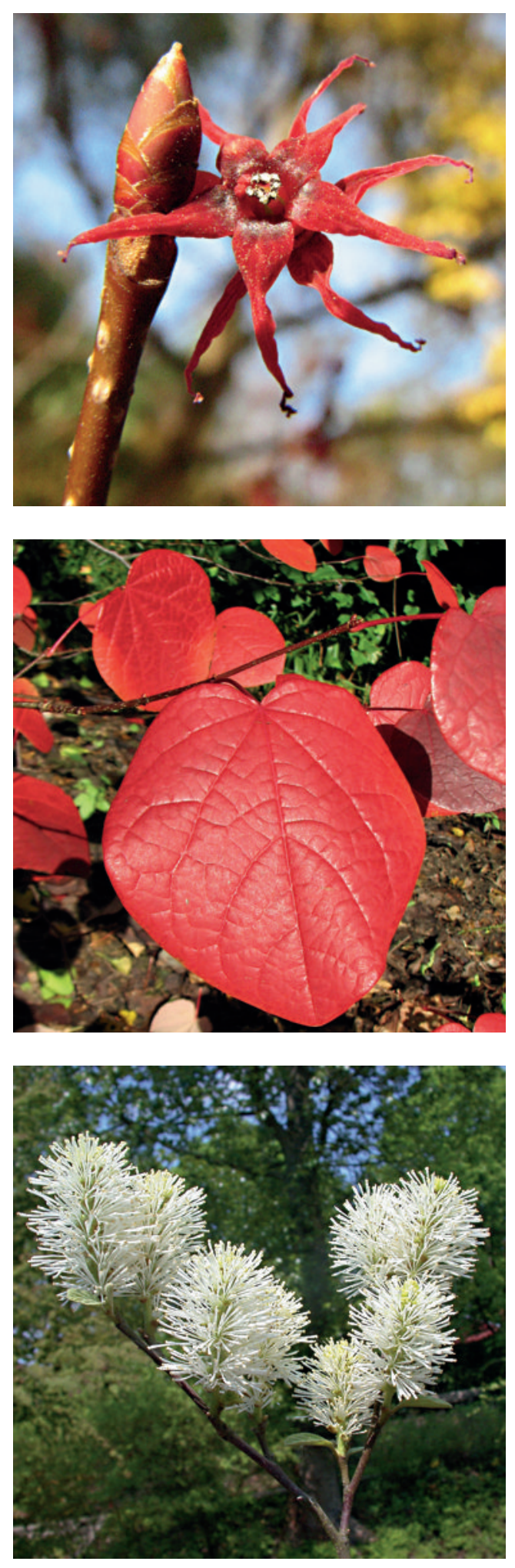

Stellung die ganzrandigen, bis $12 \mathrm{~cm}$ langen Blätter mit deutlich herzförmiger Basis. Sie ähneln denen der Judasbäume (Cercis). Oberseits sind sie glänzend dunkel- bis blaugrün, unterseits heller. Der Strauch hat eine spektakuläre farbintensive Herbstfärbung. Die Blätter färben sich dann von Orange nach Dunkel- bis Scharlachrot, mitunter auch Pflaumenblau oder Violett. Auf Standorten mit alkalischer Bodenreaktion fällt die Herbstfärbung in der Regel deutlich schwächer aus und ist meist nur fahl gelb (wie auch z. B. bei Acer rubrum oder auch Cercidiphyllum japonicum). Besonders reizvoll ist die Doppelblüte in Kombination mit gelbfärbenden Gehölzen (z. B. Acer capillipes oder Acer shirasawanum) oder auch weißblühenden Stauden wie Aster ericoides. In Kombination mit immergrünen Laubgehölzen und Koniferen oder vor hellen, ruhigen Fassaden kommt der Strauch gut zur Geltung. In der Planung muss beachtet werden, dass die Herbstfärbung bei dieser Art nur 2 bis 3 Wochen dauert. Die Blüten sind sehr unscheinbar und werden leicht übersehen. Die zwittrigen Blüten sind fünfzählig. Ein kurzer, grünlicher Kelch umgibt 5 rotbraune Kelchblätter, die an der Basis Nektardrüsen zur Beköstigung der Blütenbesucher haben. Der Fruchtknoten ist zweifächerig und enthält mehrere Samenanlagen. Die $1,5 \mathrm{~cm}$ langen, herzförmigen, braunschwarzen Kapselfrüchte reifen im Spätsommer nach der Blüte, entwickeln sich aber in Kultur eher selten. Auch diese Art gewann aufgrund des hohen Zierwertes bereits mehrere Gartenpreise.

\subsection{Fothergilla (Federbuschstrauch)}

Früher wurden vier Arten zur Gattung Fothergilla gestellt, von denen jedoch nur noch die nachfolgenden zwei allgemein anerkannt werden (KRÜSSMANN 1977). Federbuschsträucher wurden nach dem englischen Arzt JoHN FoTHERGILL (1712-1780) benannt. Die ausschließlich strauchartigen, winterkahlen Arten stammen aus den südöstlichen USA, wo sie saure Böden be-

Abb. 2 (oben): Disanthus cercidifolius.

Abb. 3 (Mitte): Laub von Disanthus cercidifolius.

Abb. 4 (unten): Fothergilla gardenii. 
siedeln. Sie sind winterkahl, echte Winterknospen fehlen. Den Schutz des Vegetationspunktes übernehmen kleine, filzig behaarte Laubblätter. Die wechselständigen Blätter sind kurz gestielt. Alle Arten zeigen eine leuchtend gelbe, orangerote bis rote Herbstfärbung. Die zwittrigen Blüten stehen in aufrechten Ähren, die bis $8 \mathrm{~cm}$ lang werden können. Die Blütenhülle ist einfach mit nur Kelchblättern, die Kronblätter fehlen. Auffallend sind die zahlreichen weißen Staubblätter mit deutlich verdickten Enden. Sie ragen weit aus den Einzelblüten hervor. Zum Blütezeitpunkt verströmen die Federbuschsträucher einen intensiven süßlichen Duft. Die sich zweiklappig öffnenden Kapseln sind bis 1,5 cm lang und borstig behaart. Für ein gesundes Wachstum, einen prächtigen Blütenflor sowie eine intensive Herbstfärbung ist ein saures bis schwach saures Substrat unerlässlich, da die Art auf Kalk in der Regel mit Kümmerwuchs reagiert. Schwere tonige oder lehmige Substrate führen ebenfalls zu einer raschen Vergreisung und einem stark verminderten Blütenflor. Der weiße Blütenaspekt der Federbuschsträucher wird durch benachbarte Gehölze mit rotlaubigem Austrieb bzw. roter Laub- bzw. Blütenfarbe besonders hervor gehoben.

Fothergilla gardenii (Erlenblättriger Federbuschstrauch) ist von North Carolina bis in den Süden von Alabama heimisch und wird meist nicht höher als 1,2 m. Im Alter ist der Strauch meist deutlich breiter als hoch und treibt gelegentlich Ausläufer. Die verkehrt-eiförmigen oder auch rundlichen, bis $4 \mathrm{~cm}$ langen Blätter sind in der oberen Hälfte unregelmäßig gezähnt und erinnern an die Blätter von Erlen (dt. Name!). Die Herbstfärbung ist leuchtend orangerot. Die bis zu 3,5 cm langen, aufrechten, flaschenbürstenartigen Ähren erscheinen vor oder mit dem Laubaustrieb von April bis Mitte Mai.

Fothergilla major (Großer Federbuschstrauch) stammt aus den Allegheny Mountains in den westlichen Appalachen. Das Gehölz wird bis $3,5 \mathrm{~m}$ hoch und verzweigt sich dicht. Seine Blätter werden bis $12(-15) \mathrm{cm}$ lang und sind breiteiförmig, auf der Unterseite leicht bereift und schwach behaart. Die Herbstfärbung reicht von Gelb über Orange bis Rot. Die Ende April bis Ende Mai erscheinenden aufrechten Blütenähren sind mit bis zu $7 \mathrm{~cm}$ Länge fast doppelt so groß wie bei der vorigen Art. Auch Fothergilla major gilt als besonders gartenwürdig.

\subsection{Hamamelis (Zaubernuss)}

Hamamelis ist die namensgebende Gattung für die Hamamelidaceae. Sie umfasst 4 Arten aus dem östlichen N-Amerika und östlichen Asien (Mabberley 2008). Hamamelis konnte bereits im Tertiär Spitzbergens mit Hamamelis clarus auch fossil nachgewiesen werden (LEHMANN et al. 1978). Zaubernüsse sind winterkahle Sträucher ohne echte Winterknospen. Im Sommer fast unscheinbar, fallen diese Pflanzen im Herbst und Winter umso mehr auf. Im Herbst beeindrucken sie durch eine spektakuläre Laubfärbung von Gelb über kräftig Orange bis hin zu leuchtend Rot. Im Winter und zeitigen Frühjahr bringen die meisten Arten ihre leuchtend gefärbten Blüten hervor (H. x intermedia: Goldgelb, H. japonica: Gelb bis Orange-Rot, H. mollis: Goldgelb mit rötlicher Basis, H. vernalis: Hellgelb mit rötlicher Basis). Die meist sehr angenehm duftenden Blüten stehen in kurz gestielten seitlichen Köpfchen. Die zwittrigen Blüten sind 4-zählig mit ausdauernden Kelchblättern, während die streifenartigen Kronblätter unmittelbar nach der Blütezeit abgeworfen werden. Letztere sind in der Knospe eingerollt und daher nach der Entfaltung stark geknittert. Die Blüten haben 4 Staubblätter und 2 Griffel. Im Vergleich zu anderen Winterblühern wie z. B. Lonicera purpursii, Jasminum nudiflorum oder Chimonanthus praecox vertragen die Blüten der Zaubernüsse deutlich mehr Frost. Bei tiefen Frösten rollen sich die Kronblätter mehr oder weniger stark zusammen. Wird es wieder milder, entrollen sich diese wieder. Der Fruchtknoten ist mittelständig. Die Früchte sind zweifächerig und weisen 1 bis $2 \mathrm{~cm}$ lange stark zurückgebogene Griffelreste auf. Die Kapseln öffnen sich zum Zeitpunkt der Samenreife explosionsartig und schleudern die Samen dementsprechend weit aus der Frucht hinaus. Das Aufknacken ist bei trockener Witterung deutlich hörbar. 

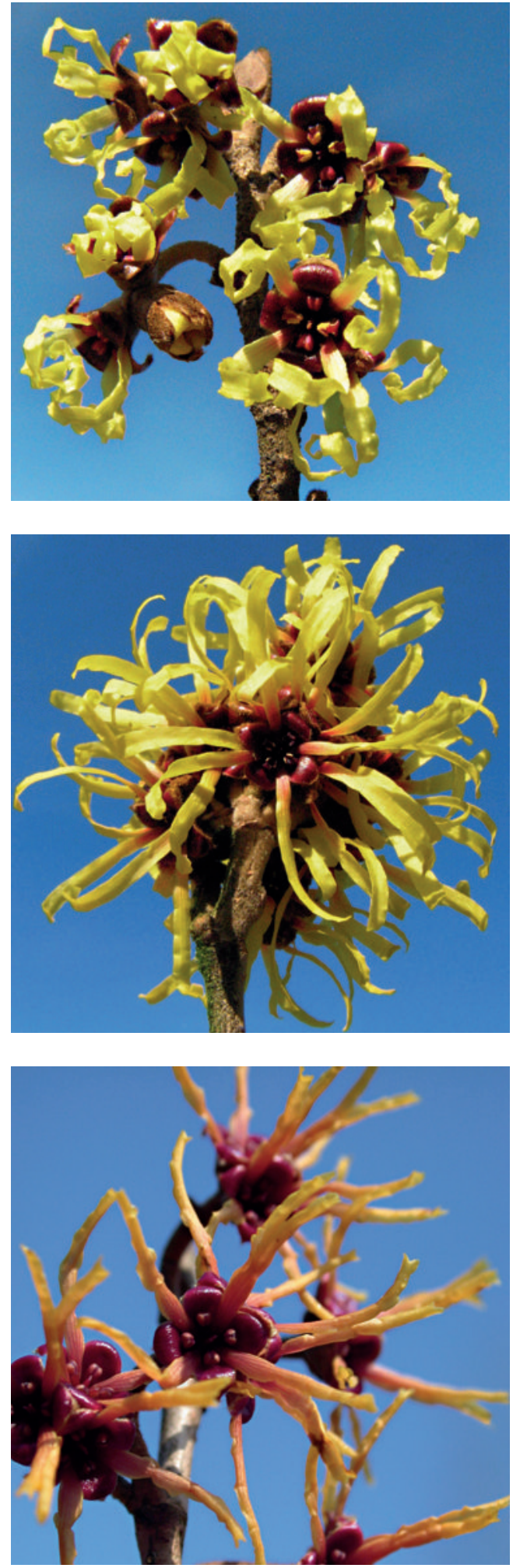

Der Blütenflor sowie die intensive Herbstfärbung der Zaubernüsse kommt vor ruhigen, dunklen Hintergründen aus Koniferen (z. B. Schnitthecke aus Taxus) oder in Kombination mit Rhododendren sowie über dunklen Bodendeckern (z. B. Hedera oder Pachysandra) besonders gut zur Geltung. Für ein optimales Gedeihen benötigen Zaubernüsse ein sandiglehmiges bis sandig-humoses, frisch bis feuchtes Substrat in sonniger bis lichtschattiger (bis halbschattiger) Lage. Staunässe im Wurzelraum wird generell nur sehr schlecht vertragen.

Hamamelis japonica (Japanische Zaubernuss) stammt aus Japan. Zum optimalen Gedeihen benötigt der bis $4 \mathrm{~m}$ hohe Strauch ausreichend feuchte Böden. Junge Pflanzen sind etwas frostempfindlich. Im Gegensatz zur Hybrid-Zaubernuss ( $H . \mathrm{x}$ intermedia) stehen die Zweige eher waagerecht als steil aufsteigend. Die Krone ist breit trichterförmig und ausladend. Junge Triebe sind zu Beginn leicht sternhaarig und verkahlen später. Die verkehrt-eiförmigen, bis $18 \mathrm{~cm}$ langen Blätter sind zuletzt unterseits nur noch auf den Nerven behaart. Im Herbst erscheint eine kräftig goldgelbe Laubverfärbung. Die Blüten sind zweifarbig, die Kelchblätter sind kräftig rotbraun, während die stark geknitterten Kronblätter intensiv gelb gefärbt sind. Der Blütezeitraum reicht von Anfang Januar bis Ende März. Die Blüten verströmen einen intensiven, weitstreichenden, angenehmen Duft.

Hamamelis mollis (Chinesische Zaubernuss) stammt aus den Provinzen Hubei und Jiangsu und kommt dort in Höhenlagen zwischen 1200 bis $2300 \mathrm{~m}$ vor. Der ausladende Strauch erreicht Höhen bis zu $5 \mathrm{~m}$ und wird meist breiter als hoch. Die Art reagiert sehr empfindlich gegenüber Bodenverdichtungen und ist wie die Japanische Zaubernuss in der Jugend etwas frostgefährdet. Auch sommerlicher Trockenstress

Abb. 5 (oben): Hamamelis japonica.

Abb. 6 (Mitte): Hamamelis mollis.

Abb. 7 (unten): Hamamelis vernalis. 
wird nur schlecht vertragen, was bei der Wahl des Standortes in Pflanzungen stets berücksichtigt werden muss. Die jungen Triebe sind anfänglich dicht behaart. Die großen, bis 15 (-20) cm langen Blätter sind beiderseits behaart, die Unterseite wesentlich dichter als die Oberseite. Die Herbstfärbung reicht von Gelb- über Orange- bis hin zu Rottönen. Bei den von Januar bis Ende März erscheinenden Blüten sind die Kelchblätter bräunlichrot, die Kronblätter orangegelb und an der Basis rötlich. Die Staubblätter und Griffel haben dieselbe Färbung wie die Kelchblätter. Die Art verfügt daher zum Blütezeitpunkt über eine großartige Fernwirkung. Aufgrund des hohen Zierwertes wurde dieses wertvolle Solitärgehölz bereits mehrfach prämiert. Neben der Naturform sind auch folgende Selektionen für die Gartenkultur von Bedeutung: 'Brevipetala' (Blüte dunkel orangegelb; sehr intensiv süßlich duftend); 'Goldcrest' (Blüte außen goldgelb, Basis braunrot; süßlicher Duft); 'Pallida' (Blüte leuchtend gelb; starker süßlicher Duft).

Hamamelis vernalis (Frühlings-Zaubernuss) ist in den südöstlichen USA heimisch. Der Strauch wird 3 bis $4 \mathrm{~m}$ hoch und treibt kurze Ausläufer, so dass sich langsam größere Gebüsche bilden können. Die Krone ist breit ausladend, im Alter meist deutlich breiter als hoch. Die bis zu $15 \mathrm{~cm}$ langen Blätter sind im Umriss verkehrt-eiförmig bis eilänglich. Ihre Unterseite ist meist leicht filzig behaart. Im Herbst färben sie sich lebhaft gold- bis orangegelb. Im Gegensatz zu den anderen Arten sind die Blüten der Frühling-Zaubernuss eher unscheinbar. Die Kelchblätter sind rötlich, die kurzen, bis $1,5 \mathrm{~cm}$ langen Kronblätter hell zitronengelb und an der Basis dunkelrot gefärbt. Die Blüten duften stark und erscheinen von Januar bis Mitte März. Eine interessante Sorte ist die Selektion 'Sandra' mit einem pflaumenblauen bis violetten Austrieb, der jedoch später vergrünt. Die Herbstfärbung ist orange bis karminrot.

Abb. 8: Hamamelis virginiana.

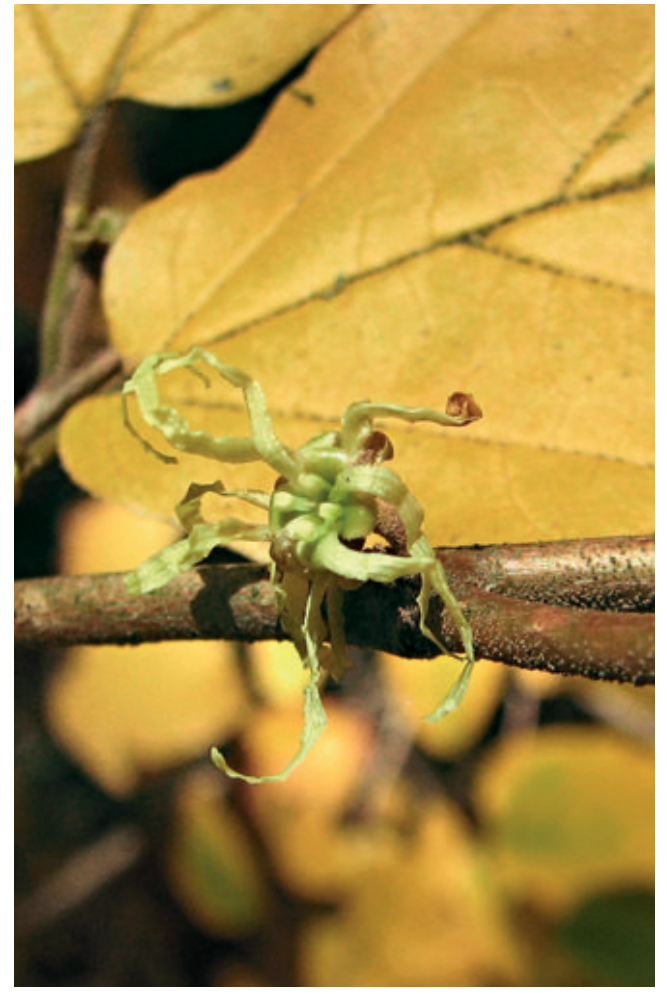

Hamamelis virginiana (Virginische Zaubernuss) ist ein 5-6 m hoher aufrechter Großstrauch aus dem östlichen Nord-Amerika, der im Unterwuchs von Wäldern und Gebüschen vorkommt. Man findet die Art auch auf nährstoffreichen Böden in der Nähe von Flussufern. Dementsprechend intolerant ist diese Zaubernuss gegenüber Trockenstress. Die $12(-15) \mathrm{cm}$ langen Blätter sind fast kahl und nur auf den Nerven der Blattunterseite behaart. Sie wurden früher von den amerikanischen Ureinwohnern als Arznei verwendet (DÖRKEN \& HÖGGEMEIER 2009). Auch heutzutage finden Extrakte aus Blättern sowie der Borke immer noch aufgrund ihrer adstringierenden Wirkung Verwendung in medizinischen und kosmetischen Produkten. Pharmazeutisch wirksame Inhaltsstoffe sind neben den glykosidischen Gerbstoffen (Hamamelitannin) weitere Gerbstoffe sowie ätherische Öle. Extrakte werden in Salben oder Tinkturen verarbeitet, die in der Wundbehandlung, der Venenpflege und zur Bekämpfung von Entzündungen im Rachenraum eingesetzt werden 
(PAHLOW 1979). Im Gegensatz zu den übrigen Arten erscheinen die hell- bis zitronengelben Blüten kurz vor oder mit dem Laubabwurf von September bis Oktober und haben daher weniger optische Fernwirkung. Sie verströmen einen deutlich wahrnehmbaren angenehmen Duft, der zahlreiche Insekten anlockt.

Hamamelis $x$ intermedia (Hybrid-Zaubernuss) wird $4 \mathrm{~m}$ hoch und ist eine Kreuzung zwischen $H$. japonica und H. mollis. Der Habitus der Hybrid-Zaubernuss ist aufgrund der aufsteigenden Äste breit trichterförmig. Die bis $15 \mathrm{~cm}$ langen Blätter sind unterseits leicht behaart und färben sich im Herbst leuchtend gelb, orange bis rot. Die dunkel goldgelben Blüten, deren Kronblätter nur an der Spitze hin leicht gebogen sind, sind ansonsten gerade und erscheinen massenhaft von Januar bis März. Beliebte Sorten sind: 'Barmstedt Gold' (Blüte goldgelb; Herbstfärbung gelb bis orangerot); 'Carmine Red' (Blüte bronzefarben; Herbstfärbung gelb); 'Diane' (Blüte dunkelrot; Herbstfärbung karminrot); 'Feuerzauber' (Blüten dunkelrot; Herbstfärbung orange bis rot); 'Primavera' (Blüte außen hellgelb, Basis purpurrot; Herbstfärbung gelb bis orange); 'Orange Beauty' (Blüte orangegelb, großblumig); 'Ruby Glow' (Blüte kupferrot); 'Sunburst' (Blüte blassgelb, großblumig); 'Winter Beauty' (Blüte goldgelb).

\subsection{Liquidambar (Amberbaum)}

$\mathrm{Zu}$ den Amberbäumen gehören 5 winterkahle Arten, die im südöstlichen N-Amerika bis Mittel-Amerika, dem östlichen europäischen Mittelmeerraum sowie in Ost-Asien heimisch sind (ENDRESS 1993). Die fossile Art Liquidambar europaea war ehemals auch in Mittel-Europa heimisch (Buecheler 2003, SCHWEIGERT 2003), sie gilt als Vorläufer des rezenten nordamerikanischen Amberbaums (Liquidambar styraciflua, Kramer 1974). In Mittel- Europa starb Liquidambar während der letzten Eiszeiten aus, so dass ein sehr disjunktes Verbreitungsareal entstand. Die botanische Bezeichnung leitet sich von lat. liquidus = flüssig und von arab. ambar $=$ Amber ab. Wie die Übersetzung vermuten lässt, handelt es sich bei den Amberbäumen um sehr harzrei- che Gehölze. Nach Verletzungen tritt schnell ein angenehm duftendes Harz aus, das als Storax bezeichnet wird. Das begehrte Harz wird im Frühjahr durch künstlich herbeigeführte Stammverletzungen gewonnen. Besonders harzreich sind Splintholz und Rinde. Das Harz von Liquidambar styraciflua (engl. sweet gum oder red gum) wurde von den nordamerikanischen Indianern als Kaugummi, aber auch in der Heilkunst verwendet. Auch heute noch ist es ein Rohstoff zur Herstellung von Kaugummis, zur Parfümierung von Seifen, Kosmetika sowie Tabakmischungen. Pharmazeutisch wird es unter anderem gegen Halsschmerzen, Hauterkrankungen oder bei Diarrhoe genutzt. Amberbäume sind winterkahle Großsträucher oder Bäume. Einige Arten wie Liquidambar styraciflua weisen an den Trieben deutliche Korkleisten auf. Die breit eiförmigen, grünen, rötlich überlaufenden Winterknospen sind kahl und glänzend. Die 3-5 (-7)lappigen Blätter erinnern an Ahornblätter, sind jedoch aufgrund ihrer wechselständigen Anordnung eindeutig von jenen zu unterscheiden. Die ab einem Alter von 20 Jahren hervorgebrachten Blüten stehen in kugeligen, endständigen Köpfchen. Die männlichen und weiblichen Blüten befinden sich räumlich voneinander getrennt auf dem selben Individuum. Den männlichen und weiblichen Blüten fehlen die Kelch- und Kronblätter. Die Bestäubung erfolgt durch Wind und die Kapseln enthalten zahlreiche kleine 4-5 mm lange geflügelte Samen.

Liquidambar styraciflua (Amerikanischer Amberbaum) stammt aus dem südöstlichen NordAmerika und ist am Naturstandort mit Acer rubrum, Alnus americana, Carya ovata, Liriodendron tulipifera, Platanus occidentalis oder auch Quercus rubra vergesellschaftet (SCHÜTT et al. 2002). Beim Amerikanischen Amberbaum handelt es sich um eine Baumart, die 20-30 (-45) m hoch wird, in Mittel-Europa meist aber deutlich kleiner bleibt. Die Art ist in der Jugend etwas frostgefährdet, so dass die Triebspitzen geschädigt werden können. Für ein optimales Gedeihen sind gut nährstoffversorgte, frische bis feuchte, durchlässige Böden mit saurer bis neutraler Bodenreaktion zu empfehlen. Auf zu 
schweren oder verdichteten Substraten vergreist die Art recht schnell. In der Regel wird jeder Gartenboden problemlos vertragen. Die Äste sind entweder vollständig korkig oder weisen mindestens deutliche Korkleisten auf. Das harte, jedoch leicht zu bearbeitende Holz mit seinem helleren Splintholz und dem dunkleren, rötlichen bis rotbraunen Kernholz wird vielfach im Möbelbau wie auch für Bau- und Konstruktionszwecke verwendet. Auch zur Herstellung hochwertiger Papierprodukte wird es genutzt. Mittlerweile ist der Amerikanische Amberbaum aufgrund der großen Nachfrage seines vielseitig verwertbaren Holzes nach der Eiche die Baumart mit dem zweithöchsten Einschlag in N-Amerika (SCHÜTT et al. 2002). Die Blätter des Amerikanischen Amberbaums werden bis $20 \mathrm{~cm}$ lang und sind deutlich 5- bis 7-lappig. Der Blattrand ist gesägt. Die spektakuläre Herbstfärbung gehört zu den intensivsten, die man kennt. Das Laub färbt sich in ein leuchtendes Gelb, Orange, Dunkelrot bis Pflaumenblau. Die kahlen Winterknospen sind glänzend grün bis grünlich rot gefärbt. Der Blütezeitraum reicht von Mitte April bis Mai. Die bis $4 \mathrm{~cm}$ breiten Fruchtstände sind bis zu $7 \mathrm{~cm}$ lang gestielt und ähneln vom Weiten denen der Platanen. Die Früchte sind zweiklappige, durch die bleibenden 2 Griffel geschnäbelte und stachelige Kapseln, die zahlreiche kleine, geflügelte, 3-5 mm lange Samen enthalten. Auch diese Art wird wegen des hohen herbstlichen Zierwertes für Parks und Gärten empfohlen. Gelegentlich werden im Baumschulhandel panaschierte Sorten wie 'Aurora' (gelb), 'Silver King' (weiß) oder 'Variegata' (gelb) sowie die Hängeform 'Pendula' angeboten.

Liquidambar orientalis (Orientalischer Amberbaum) stammt aus Kleinasien. Mit Endhöhen meist zwischen 12 und $15 \mathrm{~m}$ bleibt er deutlich kleiner als Liquidambar styraciflua. Häufig

Abb. 9 (oben): Liquidambar styraciflua, männlicher Blütenstand.

Abb. 10 (Mitte): Liquidambar styraciflua, weiblicher Blütenstand.

Abb. 11 (unten): Herbstlaub von Liquidambar styraciflua.
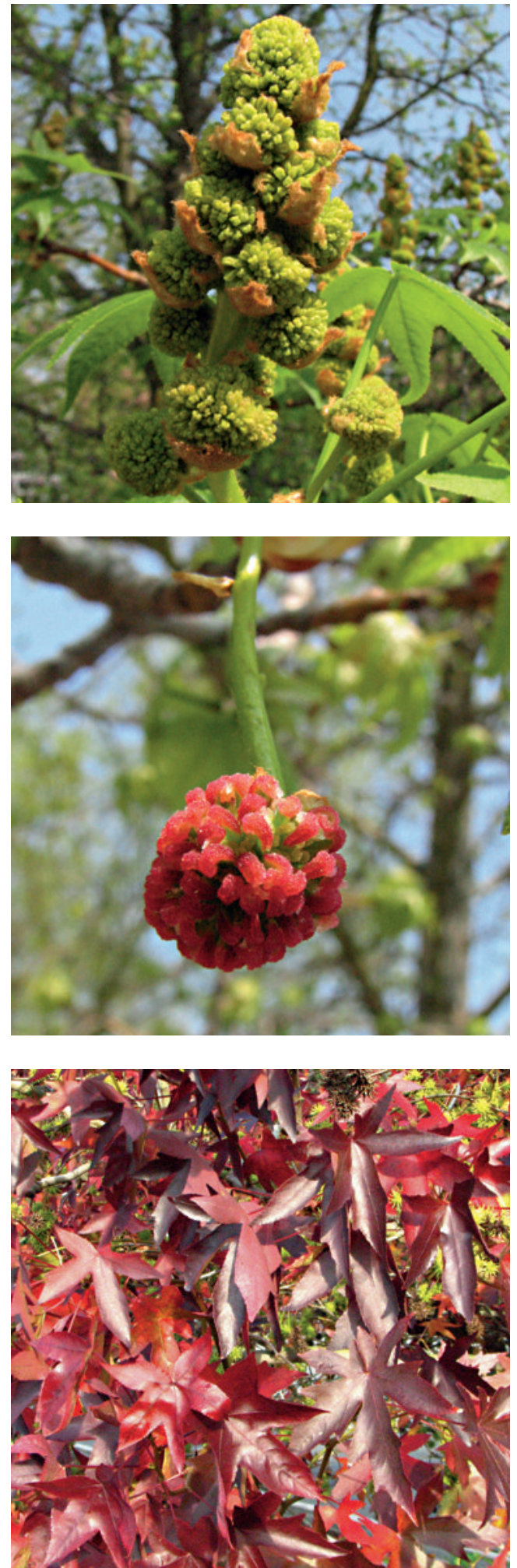
nimmt er die Wuchsform eines Großstrauches ein. Die Frosthärte liegt deutlich unter der des amerikanischen Verwandten, weswegen L. orientalis bei uns als Ziergehölz keine allzu große Rolle spielt. Das Splintholz und die Rinde sind bei dieser Art ebenfalls sehr harzreich. Die bis $10 \mathrm{~cm}$ langen Blätter sind 5-lappig. Dabei sind die drei mittleren Blattlappen ebenfalls gelappt, die Basallappen teilweise gelappt oder ungelappt. Mit einem Farbspektrum von Gelb über Orange bis Dunkelrot ist die Herbstfärbung ebenfalls spektakulär. Die Blüten erscheinen im April. Die Früchte sind denen des Amerikanischen Amberbaumes sehr ähnlich, jedoch etwa um ein Drittel kleiner.

\subsection{Parrotia persica (Parrotie, Eisenholzbaum)}

Dem deutschen Arzt, Naturforscher und Reisenden F. W. PARROT (1792-1841) zu Ehren erhielt diese Gattung ihren Namen. Der aus dem
Nord-Iran und Vorderasien stammende, winterkahle Eisenholzbaum ist ein typisches Element der kaspischen Niederungswälder. Die Gattung Parrotia konnte fossil mit P. fagifolia, die als Vorfahr von $P$. persica gilt, auch im Obermiozän Mittel-Europas nachgewiesen werden (KRAMER 1974). Bäume dieser Art werden 10-12 m hoch. Im Gegensatz zu allen anderen Zaubernussgewächsen verträgt der Eisenholzbaum auch Kalkböden. Häufig wächst er als mehrstämmiger Großstrauch, der im Alter deutlich breiter als hoch wird. Seine Borke blättert dann in mehr oder weniger großen Platten ab. Junge Triebe sind zunächst behaart und verkahlen erst später. Der Baum hat keine echten Winterknospen. Den Schutz des Vegetationspunktes übernehmen kleine, stark hellbeige behaarte Laubblätter. Die wechselständigen, verkehrt-eiförmigen Blätter werden bis $15 \mathrm{~cm}$ lang und sind beiderseits sternhaarig. Im Austrieb sind sie meist orange bis rötlich gefärbt, vergrünen jedoch relativ rasch. Der

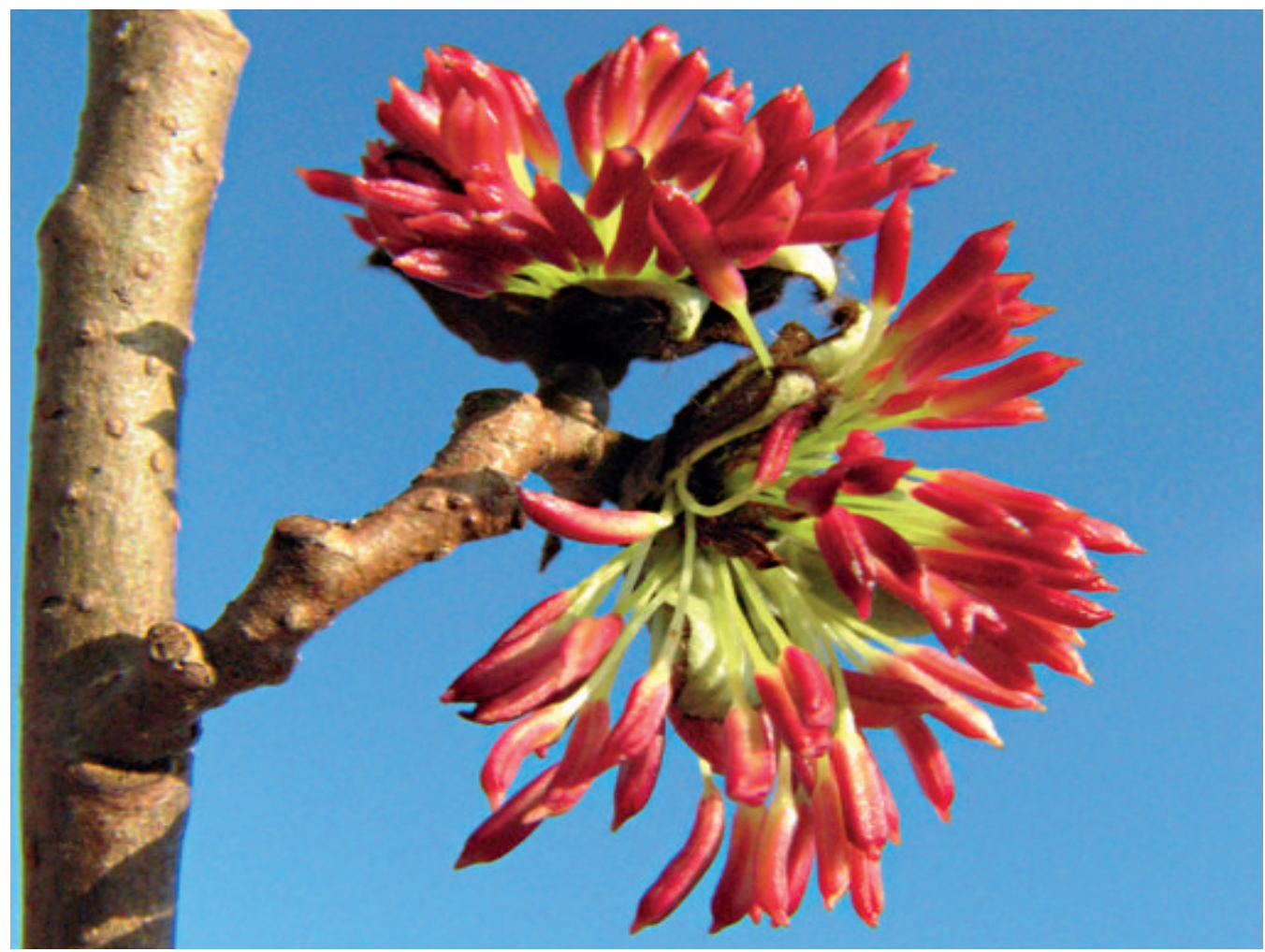

Abb. 12: Parrotia persica. 
Blattrand ist im oberen Drittel gezähnt und erinnert an die Belaubung einiger Zaubernüsse. Die lang andauernde, spektakuläre Herbstfärbung beginnt im Spätsommer bzw. im Frühherbst und ist intensiv gelb, orange, rot und violett. Parrotien haben zwittrige und rein männliche Blüten, die in dichten Büscheln stehen. Sie erscheinen von Anfang März bis Mitte April. Dabei sind die Kelchblätter klein und bräunlich rot gefärbt. Kronblätter fehlen. Die bis $2 \mathrm{~cm}$ langen, zahlreichen Staubblätter hängen weit aus der Blüte heraus, wobei die Staubbeutel leuchtend rot gefärbt sind. Der Fruchtknoten ist zweifächerig. Aus ihm entwickelt sich eine 4-klappig öffnende, bis $2 \mathrm{~cm}$ lange und dicht behaarte Kapsel. Aufgrund der bleibenden Griffel ist die Kapsel mehr oder weniger gehörnt. Wie die vorher genannten Arten wurde der Eisenholzbaum mehrfach als Pflanze mit besonderem Zierwert ausgezeichnet.

\subsection{Parrotiopsis jacquemontiana (Scheinparrotie)}

Die Scheinparrotie ist der echten Parrotie im Laub recht ähnlich, worauf wissenschaftlicher sowie deutscher Name hinweisen. Die aus dem Himalaja von Kaschmir bis Afghanistan stammenden Scheinparrotie ist ein winterkahler Großstrauch, der gelegentlich auch wie ein kleiner Baum wächst und selten Höhen von über $6 \mathrm{~m}$ erreicht. Die Art kommt meist auf sauren bis neutralen Böden vor. Gelegentlich werden auch leicht alkalische Bodenreaktionen vertragen. Die Zweige wachsen straff aufrecht und sind in der Jugend sternhaarig. Wie auch beim Eisenholzbaum wird der Schutz des Vegetationspunktes von kleinen, stark behaarten Laubblättern übernommen. Die wechselständigen Blätter sind verkehrt-eiförmig bis rundlich und bis $7 \mathrm{~cm}$ lang und erinnern an Erlenlaub. Der Blattrand ist in der Regel stark gesägt. Im Austrieb sind beide Blattseiten behaart, später meist nur noch die Blattnerven der Unterseite. Die Herbstfärbung ist gelb, orange bis rot. Von Anfang April bis Mitte Mai erscheinen kurz vor oder mit dem

Abb. 13: Parrotiopsis jacquemontiana.

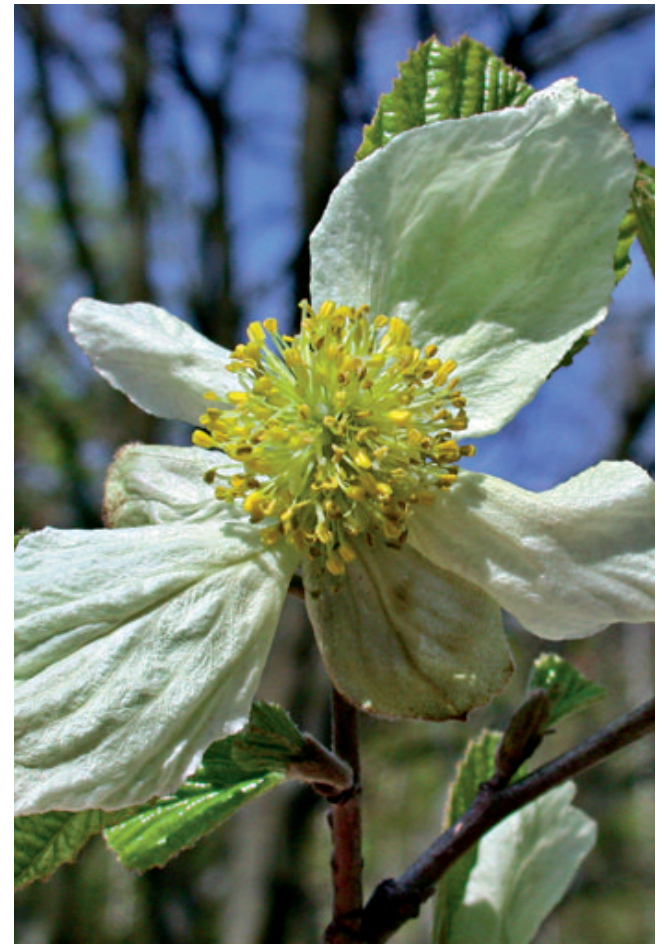

Laubaustrieb zwittrige Blüten, die einen angenehmen Duft verströmen. Die Kelchblätter sind stark reduziert, Kronblätter fehlen. Jede Blüte hat 15 gelbe bis gelblichgrüne Staubblätter und zwei Fruchtknoten. Die Einzelblüten sind unscheinbar. Sie stehen jedoch in einem bis $3 \mathrm{~cm}$ breiten Köpfchen, das von mehreren weißen, bis $3 \mathrm{~cm}$ langen, abstehenden Hochblättern umgeben ist, welche dem Blütenstand seine attraktive Fernwirkung verleihen. Entsprechendes findet man z. B. auch bei der Gruppe der Blumenhartriegel (z. B. Cornus florida oder C. kousa) sowie beim Taschentuchbaum (Davidia involucrata). Die Früchte sind bis $2 \mathrm{~cm}$ lange, sich 4-klappig öffnende, gehörnte Kapseln.

\subsection{Sycopsis sinensis (Sycopsis)}

Zur Gattung Sycopsis werden 2-3 (ENDRESS 1993) oder 5 (ERHARD et al. 2008) immergrüne Arten aus O- bis SO-Asien gezählt, von denen nur eine, Sycopsis sinensis, in milderen Lagen Mittel-Europas im Freiland ausreichend frosthart ist. Die Art stammt aus Mittel- und West-China. Sie kann auf frischen bis feuchten, neutralen bis 


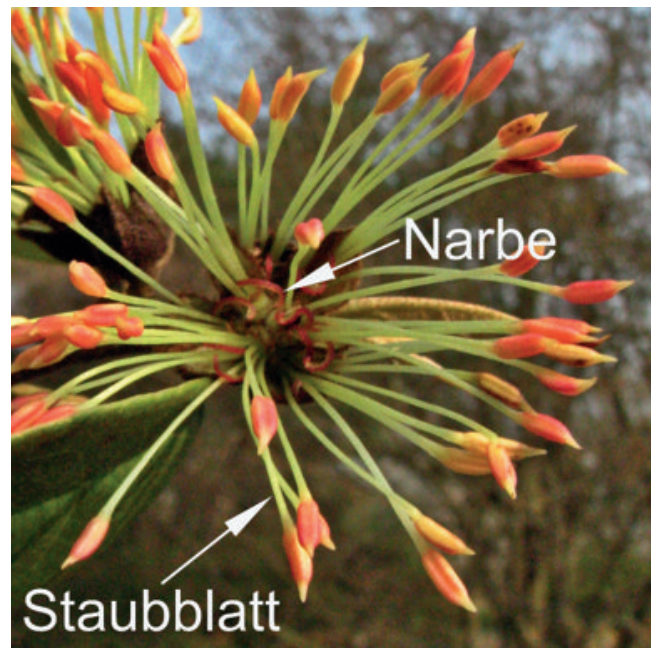

schwach sauren Standorten in windgeschützten Lagen erfolgreich kultiviert werden. Es handelt sich um immergrüne, mehrstämmige Sträucher oder kleine Bäume, die bis $6 \mathrm{~m}$ hoch werden. Junge Triebe sind anfangs meist dicht behaart, echte Winterknospen fehlen. Der Vegetationspunkt wird von kleinen, stark behaarten Laubblättern übernommen. Die wechselständig angeordneten Blätter sind derb ledrig und werden bis zu $7 \mathrm{~cm}$ lang. Ihre Form ist vielgestaltig und reicht von eiförmig-ganzrandig bis eilänglich oder elliptisch-länglich mit einem leicht gezähnten Blattrand. Die Blattoberseite ist dunkel olivgrün, die Unterseite ist wesentlich heller. Der Blattstiel ist dicht warzig besetzt. Die zwittrigen sowie männlichen Blüten erscheinen von Anfang Februar bis März, gelegentlich auch schon Ende Dezember. Ihre 5-6 Kelchblätter sind klein und recht unauffällig, Kronblätter fehlen. Bei den sich öffnenden Blütenknospen erscheinen zunächst die Staubblätter, die bis zu $2 \mathrm{~cm}$ lang werden und im voll aufgeblühten Stadium weit aus der Blüte herausragen. Ihre Staubbeutel sind kräftig orangerot gefärbt. Die hingegen eher unscheinbaren und daher leicht zu übersehenden, stark gekrümmten Narben sind dunkelrot und glänzend. Die braunen Kapselfrüchte sind aufgrund der bleibenden Griffel geschnäbelt und werden bis $2 \mathrm{~cm}$ lang. Sie enthalten 2 Samen. Diese Art wird meist nur von Sammlern und in botanischen Gärten kultiviert.

\section{Literatur}

DIRR, R. 1994: Hamamelis und andere Zaubernussgewächse. - Stuttgart.

Dörken, V.M. \& Höggemeier, A. 2009: Botanischdendrologische Streifzüge. Gehölzführer des Botanischen Gartens Bochum. - Bochum.

ENDRESS, P. K. 1993: Hamamelidaceae. In: KUBITZKI, K.: The families and genera of vascular plants. II,

Flowering plants, Dicotyledons: Magnoliid, Hamamelid and Caryophyllid families. - Heidelberg.

ERhardt, W., GötZ, E., BÖdeCKer, N. \&

Seybold, S. 2008: Der große Zander. - StuTtGart.

Ferguson, D. K. \& KNOBLOCH, E. 1998:

A fresh look at the rich assemblage from the Pliocene sink-hole of Willershausen, Germany. - Rev. Palaeobot. Palyn. 101: 271-286.

FukareK, F. 2000: Urania Pflanzenreich. - Berlin. Lehmann, U., Thiedig, F. \&. Harland, W. B. 1978: Spitzbergen im Tertiär. - Polarforschung 48: 120-138. Kelly, J. \& Hillier, J. 2004: Bäume und Sträucher, 2. Aufl. - Braunschweig.

Kramer, K. 1974: Fossile Pflanzen aus der Braunkohlezeit: Die obermiozäne Flora des unteren Fischbachtones im Tagebau Frechen bei Köln. -

Mitt. Dtsch. Dendrol. Ges. 67: 199-233.

KRÜSSMANN, G. 1976-1978: Handbuch der Laubgehölze, 2. Aufl. - Berlin, Hamburg.

Mabberley, D. J. 2008: Mabberley's plant book,

3. Aufl. - Cambridge.

MAI, D. H. 1995: Tertiäre Vegetationsgeschichte Europas, Methoden und Ergebnisse. - Jena.

ENgLeR, A. 1964: Syllabus der Pflanzenfamilien,

2. Band. Angiospermen. 12. Aufl. - Berlin.

SCHMIDT, P. 2005: Bäume und Sträucher Kaukasiens -

Teil IV. Laubgehölze der Familien Globulariaceae (Kugelblumengewächse) bis Punicaceae (Granatapfelgewächse). - Mitt. Dtsch. Dendrol. Ges. 90: 25-44. SchÜTt, P., Schuck, H. J. \& Stimm, B. 2002: Lexikon der Baum- und Straucharten. - Hamburg. PAHLOW, M. 1979: Das große Buch der Heilpflanzen. München.

TAYlOR, T. N., TAYlor, E. L. \& KRINGS, M. 2009: Palaeobotany: The biology and evolution of fossil plants. Oxford.

Abb. 14: Sycopsis sinensis. 